\title{
Suivi Thérapeutique Pharmacologique Des Antituberculeux : Quinze Ans d'Expérience
}

\section{Omaima El Bouazzi, PhD Student}

Centre Anti Poison et de Pharmacovigilance du Maroc- Rabat-Maroc

Faculté des sciences, Universités Ibn Tofail- Kénitra-Maroc

\section{Soufiane Belabbes, Physician, PhD Student}

Service d'imagerie médicale, Hôpital Militaire- Rabat-Maroc

Faculté des sciences, Universités Ibn Tofail- Kénitra-Maroc

\section{Latifa Ait Moussa, PhD}

Centre Anti Poison et de Pharmacovigilance du Maroc- Rabat-Maroc

\section{Rachida Soulaymani-Bencheikh (Prof. of pharmacology)}

Centre Anti Poison et de Pharmacovigilance du Maroc- Rabat-Maroc

Faculté de médecine et de pharmacie,

Universités Mohammed V- Rabat-Maroc

\section{Narjis Badrane, Physician, PhD Student}

Centre Anti Poison et de Pharmacovigilance du Maroc- Rabat-Maroc

Faculté des sciences, Universités Ibn Tofail- Kénitra-Maroc

Rachid Bengueddour, Prof. of biology

Faculté des sciences, Universités Ibn Tofail- Kénitra-Maroc

\section{doi: 10.19044/esj.2016.v12n21p35 URL:http://dx.doi.org/10.19044/esj.2016.v12n21p35}

\begin{abstract}
Tuberculosis is a world public health problem. Its treatment is based on a set of drugs that can cause many side effects. Liver toxicity remains one of the most common and severe side effect causing treatment failure. The objective of this study is to demonstrate the interest of therapeutic drug monitoring in the treatment of tuberculosis through the experience of the Laboratory of Toxicology and Pharmacology of the Anti Poison Center. This retrospective study involved 1152 patients who had benefited from a determination of plasma levels of first line antitubureculosis drugs (isoniasid, pyrazinamid, rifampicin) during 15 years. 135 patients had developed hepatotoxicity. The study showed that at therapeutic dose, $71.2 \%$ of TB patients had plasma concentrations lower than the therapeutic range, and $57.8 \%$ of patients had isoniazid plasma concentrations above the therapeutic range. This interindividual variability justifies the need for therapeutic drug monitoring to assess an inadequate response to tuberculosis treatment and
\end{abstract}


prevent the development of hepatotoxicity.

Keywords: Antituberculosis, Therapeutic drug monitoring

\section{Résumé}

La tuberculose est un problème de santé publique dans le monde entier. Son traitement se base sur un ensemble de médicaments qui peuvent être à l'origine de nombreux effets indésirables. L’hépatotoxicité reste la plus fréquente et la plus redoutable, pouvant parfois conduire à un échec thérapeutique. L'objectif de cette étude est de montrer l'intérêt du suivi thérapeutique pharmacologique des antituberculeux à travers l'expérience du Laboratoire de Toxicologie et de Pharmacologie du Centre Anti Poison et de Pharmacovigilance du Maroc. Il s‘agit d’une étude rétrospective étalée sur une durée de 15 ans, et menée sur 1152 patients ayant tous bénéficié d’un suivi thérapeutique pharmacologique des antituberculeux de première ligne (isoniazide, rifampicine, et pyrazinamide) et dont 135 patients avaient développé une hépatotoxicité. Notre étude a montré qu’à dose thérapeutique, 71,2\% des patients tuberculeux avaient des concentrations plasmatiques de rifampicine au dessous de la fourchette thérapeutique et 57,8\% des patients avaient des concentrations plasmatiques d'isoniazide au dessus de la fourchette thérapeutique. Cette variabilité interindividuelle justifie la nécessité d'un ajustement thérapeutique afin d’éviter l'échec thérapeutique ou la toxicité hépatique.

Mots Clés: Antituberculeux, Suivi Thérapeutique Pharmacologique

\section{Introduction}

La tuberculose (TB) est une maladie infectieuse causée par le Mycobacterium tuberculosis. Elle constitue un problème de santé publique à l'échelle internationale avec 9,6 millions de nouveaux cas enregistrés en 2014 et 1,5 millions de décès (Rapport OMS, 2014). Au Maroc, 28135 nouveaux cas de tuberculose ont été signalés en 2014, soit une incidence de 106 [97-115] nouveaux cas pour 100.000 habitants selon le rapport de l’organisation mondiale de la santé (Rapport OMS, 2014).

Le panel standard du traitement antituberculeux le plus utilisé comprend principalement quatre médicaments: L’isoniazide (INH), la rifampicine (RIF), le pyrazinamide (PZA) et l'éthambutol (ETB) (OMS guidelines, 2010 ; Blumberg et al, 2003). Ces médicaments sont connus pour leurs effets indésirables multiples, notamment hépatiques dont le mécanisme est mal connu (Yee et al, 2003 ; Tostmann et al, 2008 ; Saukkonnen et al, 2006).

L’optimisation de la posologie est une approche pluridisciplinaire 
dont l'objectif est d'améliorer la prise en charge et le bien-être du patient. De ce fait, le Suivi Thérapeutique Pharmacologique (STP) peut jouer un rôle stratégique dans l'amélioration de la prise en charge des patients par l'optimisation des doses thérapeutiques non toxiques (Peloquin, 2002).

Le principe consiste à faire des dosages plasmatiques afin de déterminer la posologie la plus adaptée permettant d’obtenir des concentrations sanguines optimales, et d'éviter l'apparition des effets indésirables dose dépendants. Dans le cadre du traitement antituberculeux, le recours au STP permet aux cliniciens d'adapter le schéma thérapeutique pour chaque patient selon le terrain et les résultats analytiques obtenus (Tostmann et al, 2008).

L’objectif de ce travail est de décrire l'expérience du Laboratoire de Toxicologie et de Pharmacologie du Centre Anti Poison et de Pharmacovigilance du Maroc (CAPM-LAB) dans le STP des antituberculeux, et de montrer l'intérêt du dosage plasmatique des antituberculeux de première ligne.

\section{Matériels et méthodes}

\section{Type d'étude et population}

Il s'agit d'une analyse rétrospective et descriptive de la base de données du CAPM-LAB de janvier 1999 à décembre 2014 (15 ans). Cette étude inclue tous les patients ayant bénéficié d'un dosage plasmatique de l'INH, de la RIF et du PZA. Les prélèvements ont été reçus à partir de différentes structures sanitaires du royaume. Chaque prélèvement est accompagné d'une fiche de prescription.

\section{Traitement des échantillons}

Les prélèvements étaient effectués 2 heures après la prise du médicament pour le dosage du PZA et de la RIF et 3 heures après la prise du médicament pour le dosage de l'INH. Les échantillons étaient ensuite acheminés au CAPM-LAB selon les conditions de transport des spécimens biologiques $\left(+4^{\circ} \mathrm{C}\right)$. Après la séparation, le plasma était conservé à $-20^{\circ} \mathrm{C}$ jusqu'au jour du dosage. Comme toute détermination quantitative, une gamme d'étalonnage était préparée avec un contrôle de qualité interne à chaque dosage. Après une extraction liquide-liquide, l'échantillon était injecté dans un chromatographe en phase liquide à détecteur UV (CLHPUV).

\section{Analyse des données}

Les résultats ont été saisis sur une base de données du CAPM-LAB. Les informations comprenaient toutes les données épidémiologiques des patients fournies par les cliniciens. Les variables étudiées étaient relatives 
aux patients : l'âge, le sexe, la forme de la tuberculose, l'indice de masse corporelle (IMC), le traitement antituberculeux et sa posologie, le motif de la demande et aux résultats des dosages (concentrations plasmatiques, interprétation selon les fourchettes thérapeutiques).

Les concentrations plasmatiques étaient exprimées en $\mu \mathrm{g} / \mathrm{ml}$. Les fourchettes thérapeutiques des médicaments dosés sont : 1-2 $\mu \mathrm{g} / \mathrm{ml}$ pour l'INH, 8-24 $\mu \mathrm{g} / \mathrm{ml}$ pour la RIF et $30-50 \mu \mathrm{g} / \mathrm{ml}$ pour le pyrazinamide.

Les variables sont représentées en moyenne \pm écart type ou en médiane et intervalle interquartile.

\section{Résultats}

Durant ces quinze années, les analyses ont concerné 1152 patients tuberculeux sous traitement, pour lesquels, 1492 analyses étaient effectuées et réparties comme suit : 945 dosages d'INH (63,3\%), 448 dosages de RIF (30\%) et 99 dosages de PZA (9,7\%).

Le sexe féminin représentait $52,8 \%$ des cas soit un sex-ratio (H/F) de 0,9. L’âge moyen des patients était de 46,2 $\pm 18,4$ ans. Les tranches d'âge des adultes et des personnes âgées étaient les plus représentées avec respectivement $84,7 \%$ et $12,4 \%$ des cas. Le poids moyen des patients était de

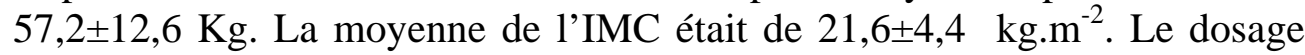
plasmatique était effectué dans un but d'adaptation posologique dans $81,4 \%$ des cas ou en cas d'apparition d'effets indésirables dans 18\% des cas. Les autres données épidémiologiques sont représentées dans le tableau 1.

Parmi les patients de notre étude, $18 \%$ avaient développé des effets indésirables dont 65,2\% avaient une hépatotoxicité, 11,1\% une intolérance digestive aux antituberculeux, 9,2\% des effets neurologiques, 7,2\% des effets cutanés, 3,9\% des troubles métaboliques, 2,9\% des troubles psychiatriques et $0,5 \%$ des troubles hématologiques (Tableau 1 ).

Tableau 1 : Caractéristiques épidémiologiques des patients sous antituberculeux ayant bénéficié d'un suivi thérapeutique pharmacologique au CAPM-LAB (N=1152).

\begin{tabular}{|c|c|}
\hline Caractéristiques & Valeurs trouvées \\
\hline Age (ans), (n=955) & $46,2 \pm 18,4$ \\
\hline Tranche d'âge, $(\mathrm{n}=957)$ & \\
\hline Enfant & $3(0,3 \%)$ \\
\hline Adolescent & $24(2,5 \%)$ \\
\hline Adulte & $811(84,7 \%)$ \\
\hline Vieillards & $119(12,4 \%)$ \\
\hline Sexe féminin (n=1152) & $608(52,8 \%)$ \\
\hline Poids (Kg), $(\mathrm{n}=927)^{*}$ & $57,2 \pm 12,6$ \\
\hline IMC (Kg.m $\left.{ }^{-2}\right),(\mathrm{n}=434)^{*}$ & $21,6 \pm 4,4$ \\
\hline Forme de la tuberculose (n=327) & \\
\hline Pulmonaire & $141(43,1 \%)$ \\
\hline Extrapulmonaire & $186(56,9 \%)$ \\
\hline Motif du dosage, (n=1152) & \\
\hline
\end{tabular}




\begin{tabular}{|c|c|}
\hline Adaptation de la posologie & $938(81,4 \%)$ \\
\hline Effet indésirable & $207(18 \%)$ \\
\hline Modification du schéma thérapeutique & $3(0,3 \%)$ \\
\hline Initiation du traitement & $4(0,3 \%)$ \\
\hline Effets indésirables, (n=207) $* *$ & $135(65,2 \%)$ \\
\hline Troubles du système hépatobiliaire & $19(9,2 \%)$ \\
\hline Troubles du système nerveux central et \\
périphérique & $23(11,1 \%)$ \\
\hline Troubles du système gastro-intestinal & $6(2,9 \%)$ \\
\hline Troubles Psychiatriques & $15(7,2 \%)$ \\
\hline Troubles de la peau et des phanères & $1(0,5 \%)$ \\
\hline Troubles hématologiques & $8(3,9 \%)$ \\
\hline Troubles métaboliques et nutritionnels & $123(15,5 \%)$ \\
\hline Traitement prescrit (n=793) ** & $400(50,4 \%)$ \\
\hline INH+RIF+PZA+ETB & $5(0,6 \%)$ \\
\hline INH+RIF & $65(8,2 \%)$ \\
\hline INH+RIF+PZA & $200(25,2 \%)$ \\
\hline RIF & INH \\
\hline$*$ Moyenne \pm écart type, ** : Effectif (pourcentage)
\end{tabular}

945 (63,3\%) dosages d'INH ont été effectués au sein du laboratoire. Les résultats des dosages montraient que $57,8 \%$ des concentrations étaient supérieures à la fourchette thérapeutique, contre $24,4 \%$ à l'intérieur de cette fourchette (figure1). La médiane des concentrations plasmatiques trouvées en $\mu \mathrm{g} / \mathrm{ml}$ était de 2,5 [1,3-4,6]. La médiane des doses prescrites en $\mathrm{mg} / \mathrm{kg} / \mathrm{j}$ était de 4,4 [3-5] (Tableau 2).

Pour le cas de la RIF, 448 dosages ont été effectués. Les résultats des dosages montraient que $71,2 \%$ des concentrations étaient au-dessous de la fourchette thérapeutique contre $27,7 \%$ à l'intérieur de cette fourchette, avec une médiane de concentrations de 5,1 [1,7-9,1] $\mu \mathrm{g} / \mathrm{ml}$ (Figure1). La moyenne des doses prises par les patients en $\mathrm{mg} / \mathrm{kg} / \mathrm{j}$ était de 9,4 [7,8-10,5] (Tableau 2).

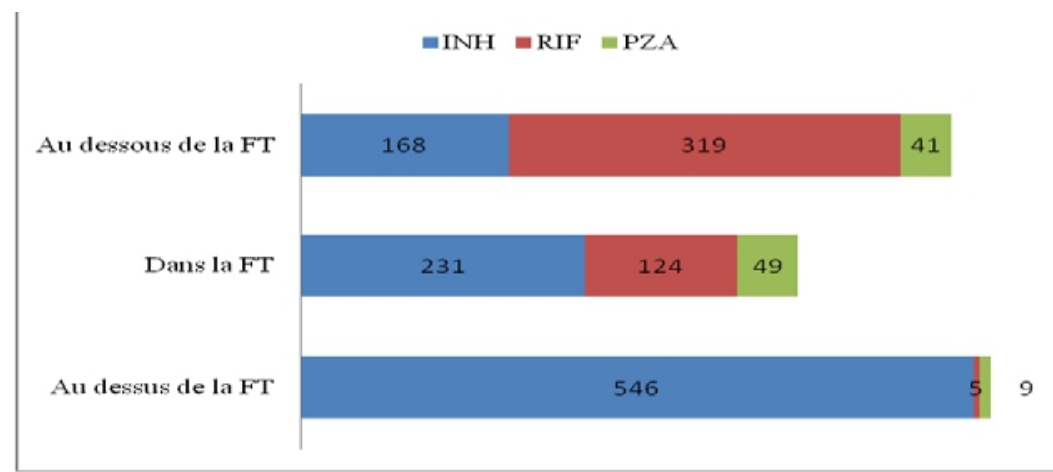

Figure 1 : Résultats des concentrations plasmatiques déterminés selon les fourchettes thérapeutiques. *FT = Fourchette thérapeutique 
Le troisième médicament dosé était le PZA avec un total de 99 dosages. Les résultats des dosages montraient que $41,4 \%$ des concentrations étaient au dessous de la fourchette thérapeutique contre 49,5\% à l'intérieur de cette fourchette (Figure1). La médiane des doses prescrites en $\mathrm{mg} / \mathrm{kg} / \mathrm{j}$ était de 22,8 [15,9-26,7] (Tableau2).

Tableau 2 : Données analytiques des trois médicaments antituberculeux

\begin{tabular}{|c|c|c|c|}
\hline Molécule dosée & $\begin{array}{c}\text { Doses administrées } \\
(\mathrm{mg} / \mathrm{kg} / \mathrm{j}) * * *\end{array}$ & $\begin{array}{c}\text { Concentrations } \\
\text { plasmatiques } \\
(\mu \mathrm{g} / \mathrm{ml}) * * *\end{array}$ & $\begin{array}{c}\text { Concentrations à } \\
\text { l'intérieure de la FT }\end{array}$ \\
\hline INH & $4,4[3-5],(\mathrm{n}=742)$ & $2,5[1,3-4,6],(\mathrm{n}=945)$ & $231(24,4 \%)$ \\
\hline RIF & $\begin{array}{c}9,4[7,8-10,5], \\
(\mathrm{n}=434)\end{array}$ & $5,1[1,7-9,1],(\mathrm{n}=448)$ & $124(27,7 \%)$ \\
\hline PZA & $\begin{array}{c}22,8[15,9-26,7], \\
(\mathrm{n}=158)\end{array}$ & $\begin{array}{c}33,8[23,3-41,5], \\
(\mathrm{n}=99)\end{array}$ \\
\hline \multicolumn{3}{|c|}{$* * *:$ Médiane [Intervalle interquartile] } \\
\hline
\end{tabular}

\section{Discussion}

De nombreuses techniques permettent la réalisation de ces dosages dont CLHP et les techniques d'immunodosage ou spectrophotométriques. Au sein de notre structure (CAPM-LAB), la mesure des concentrations plasmatiques se fait toujours par CLHP. Le choix de la technique se base sur plusieurs critères, tels que la sensibilité, la spécificité, la précision et la rapidité. Compte tenu de ces paramètres, la chromatographie est la technique analytique de référence dans le domaine pharmacologique.

L'activité du suivi thérapeutique pharmacologique des antituberculeux a commencé au CAPM-LAB en 1999 avec le développement d'une technique de dosage de l'INH par CLHP-UV. Des années après, une deuxième méthode de dosage de la RIF était validée par l'équipe du laboratoire avant de valider une troisième du PZA. Ceci explique la différence de nombre d'analyses entre les trois médicaments (INH=945 analyses, $\mathrm{RIF}=448$ analyses et $\mathrm{PZA}=99$ analyses).

Parmi les patients qui ont développé des effets indésirables et qui ont bénéficié d'un STP, 65,2\% avaient des effets hépatiques qui représentaient la tête de liste des autres effets secondaires. Le mécanisme biochimique d'hépatotoxicité aux antituberculeux et le lien entre les concentrations et l'effet hépatique ne sont pas clairement définis, mais le risque de survenue d'hépatotoxicité semble être lié au profil d'acétylation du patient (Bel Habib et al, 2006 ; Bouchentouf et al, 2011). Les facteurs génétiques ont été signalés comme étant un facteur d’hépatotoxicité dans différentes études (Huang, 2007 ; Singh et al, 2014 ; Cho et al, 2007). Ces facteurs ont été attribués à la variabilité génétique dans la capacité d'acétylation de l'INH par l'arylamine N-acetyltransferase2 (NAT2) qui est une enzyme cytosolique principalement présente dans le foie (Cho et al, 
2007). Selon les variantes du gène NAT2, trois phénotypes peuvent être définis : Les acétyleurs rapides (RA), intermédiaires (IA) et lents (SA). Dans le contexte marocain, une étude faite par l'équipe de génétique de l'institut national d’hygiène à Rabat avait étudié la variabilité génétique du gène NAT2 (Guaoua et al, 2014). Cette étude a montré que les génotypes répandus du gène NAT2 chez les Marocains sont ceux qui codent pour les SA (72,39\%), conduisant à un risque élevé d’hépatotoxicité. En terme de concentrations plasmatiques, nos résultats montrent que 63,3\% des patients avaient des concentrations d'INH au dessus de la fourchette thérapeutique ce qui confirme que les patients marocains métabolisent lentement l'INH même avec des doses inferieures à celle recommandée dans la littérature (5 mg/kg/j) (OMS guidelines, 2010 ; Rautlin et al, 1972 ; El Ftouh, 1998).

D'autre part, les concentrations plasmatiques de la RIF étaient basses dans $71,2 \%$ des cas exposant les patients à un risque élevé d'échec thérapeutique. Un prélèvement à la 6 éme heure est recommandé pour différencier entre une absorption retardée et une malabsorption. Les absorptions retardées n'exigent pas un ajustement de doses à l'inverse des malabsorptions où l'augmentation des doses administrées est fortement recommandée pour garantir une meilleure réponse thérapeutique (Peloquin, 2002).

L’absorption de la RIF est influencée d'une part par le régime alimentaire car la prise des repas gras tardent la Cmax (Tmax), et d'autre part par l'association avec les autres antituberculeux, notamment, l'INH et le PZA- qui réduit considérablement son absorption. Pour une efficacité meilleure, plusieurs auteurs proposent une dose de $1200 \mathrm{mg}$ ou plus par jour (Mitchison et al, 2000 ; Ruslami et al, 2006 ; Streingart et al, 2011 ; Acocella et al, 1989 ; Boeree et al, 2013).

Étant donné que la même dose d'un médicament ne donne pas le même effet chez tous les patients, ni la même concentration aux sites d'actions. Le recours au STP est nécessaire afin d'ajuster le régime posologique dans le but de diminuer le risque toxique des médicaments, d'atteindre l'effet thérapeutique attendu, et de diminuer le taux d'échec thérapeutique (Chast, 2004). Pour les antituberculeux, le dosage des médicaments n'est pas recommandé en routine. Cependant, il est nécessaire en cas d'échec thérapeutique non expliqué par une mauvaise observance ou une résistance du germe, en cas d'interactions médicamenteuses ou chez les patients dont l'état physiopathologique peut modifier la cinétique des antituberculeux (insuffisance rénale, insuffisance hépatique, malabsorption digestive,...) (Harcouet et al, 2007). Il est important de savoir qu'il existe une grande variabilité interindividuelle à la réponse au traitement antituberculeux, qui fait qu'une dose active chez un patient peut être toxique chez un autre. 


\section{Conclusion}

Le suivi thérapeutique pharmacologique des antituberculeux est un outil complémentaire performant pour l'ajustement posologique. Il permet d'éviter l'échec thérapeutique en cas de sous dosage et de prévenir la toxicité en cas de surdosage. L'interprétation des résultats doit être adaptée à chaque patient en raison de la grande variabilité métabolique d’origine génétique et du terrain particulier des patients.

\section{References:}

Acocella G, Bertrand A, Beytout J , et al. Comparaison of three regimens in the treatment of acute brucellosis: amulticenter multinational study. J antimicrob Chemother. 1989; 23 (3):433-439.

Al Sultan A, Peloquin CA. Therapeutic drug monitoring in the treatment of tuberculosis: An update. Drugs. 2014.

Bel Habib D, Ben Abdelghaffar H, et al. Les facteurs de risques de la toxicité hépatique des traitements antituberculeux. Revue des maladies respiratoires. 2006 ; 23 : 45-48.

Blumberg HM, Burman WJ, Chaisson RE, Daley CL, Etkind SC, Friedman LN, Fujiwara P, Grzemska M, Hopewell PC, Iseman MD, et al. American Thoracic Society: Centers for Disease Control and Prevention/Infectious Diseases Society of America: treatment of tuberculosis. Am J Respir Crit Care Med. 2003; 167: 603-62.

Boeree M, Diacon A, Dawson R, et al. What is the "right" dose of rifampicin ?. The annual conference on retroviruses and opportunistic infections; Atlanta, GA 2013.

Bouchentouf R , El jastimi S, Benjelloun A, Aitbenasser MA. Hépatotoxicité des antituberculeux : épidémiologie, mécanisme et conduite à tenir. J. Afr. Hépatol. Gastroentérol. (2011) 5:168-173.

Chast F. le suivi thérapeutique des médicaments anti-infectieux. Revue française des laboratoires. 2004 ; 365 : 21-26.

Cho HJ, Koh WJ, Ruy YJ, Ki CS, Nam MH. Genetic polymorphisms of NAT2 and CYP2E1 associated with tuberculosis drug-induced hepatotoxicity in Korean patients with pulmonary tuberculosis. Tuberculosis. 2007; 87: 551-556.

El Ftouh M, Mouline S, Badsi A, El Fassi El Fihry MT. Médicaments antituberculeux: effets secondaires et conduite à tenir. Médecine du Maghreb. 1998 ; 67: 35-38.

Guaoua S, Ratbi I, Laarabi FZ, Elalaoui SC, Jaouad IC, Barkat A, Sefiani A. Distribution of allelic and genotypic frequencies of NAT2 and CYP2E1 variants in Moroccan population. BMC Genetic. 2014; 156(15).

Harcouet L, Tod M. Intérêt du suivi thérapeutique des antituberculeux chez 
l'adulte. La lettre de l'infectiologue. 2007 ; 4 :134-142.

Huang YS. Genetic polymorphisms of drug-metalolizing enzymes and the susceptibility to antituberculosis drug-induced liver injury. Drug metab toxicol. 2007; 3(1): 1-8.

Mitchison DA. Role of individual drugs in the chemotherapy of tuberculosis. Int J tuberc Lung Dis. 2000;4(9):796-806.

Peloquin CA. Therapeutic drug monitoring in the treatment of tuberculosis. Drugs. 2002; 62: 2169-2183.

Rautlin Y, Morichau-Beauchant G, Patte F. Comparaison des taux sériques de rifampicine avec une posologie habituelle et réduite (600 et $300 \mathrm{mg} /$ jour). Médecine et maladies infectieuse.1971-1972;12:495-503.

Ruslami R, Nijland H, Aarnoutse R, et al. Evaluation of high-versus standard-dose rifampin in Indonesian patients with pulmonary tuberculosis. Antimicrob Agents Chemother. 2006; 50 (2) : 822-823.

Saukkonen JJ, Cohn DL, Jasmer RM, Schenker S, Jereb JA, Nolan CM, Peloquin CA, Gordin FM, Nunes D, Strader DB, Bernardo J, Venkataramanan R, Sterling TR. An official ATS statement: hepatotoxicity of antituberculosis therapy. Am J Respir Crit Care Med. 2006, 174:935-952.

Singh M, Gupta VH, Amarapurkar DN et al. Association of genetic variants with anti-tuberculosis drug induced hepatotoxicity: A high resolution melting analysis. Infection, genetics and evolution. 2014; 23: 42-48.

Steingart KR, Jotblad S, Robsky K, et al. Higher-dose rifampin for the treatment of pulmonary tuberculosis: a systematic review. Int J Tuberc Lung Dis. 2011; 15 (3): 305-316.

Tostmann A, Boeree MJ, Aarnoutse RE, de Lange WC, van der Ven AJ, Dekhuijzen R. Antituberculosis drug-induced hepatotoxicity: concise up-todate review. J Gastroenterol Hepatol. 2008; 23: 192-202.

World Health Organization. Global tuberculosis report 2014. Geneva: World Health Organization, 2014.

World Health Organization. Treatment of tuberculosis: guidelines. 4th ed. Geneva: World Health Organization, 2010.

Yee D, Valiquette C, Pelletier M, Parisien I, Rocher I, Menzies D. Incidence of serious side effects from first-line antituberculosis drugs among patients treated for active tuberculosis. Am J Respir Crit Care Med. 2003; 167: 14727. 